\title{
Hip pain
}

Carlos Garcia-Porrua, Miguel A Gonzalez-Gay, Juan Corredoira, Manuel Vazquez-Caruncho

\section{Clinical history}

A 34 year old man presented at the hospital because of widespread pain in his left buttock, groin and posterior thigh that had started gradually eight days earlier. He had been diagnosed with human immunodeficiency virus (HIV) disease at the age of 24 . Because of a pneumocystis carinii pneumonia he was required to be bedridden for three weeks during the month before admission. On physical examination diffuse pain to palpation on the left groin, buttock, and posterior thigh was noticed. In addition, an excruciating pain was provoked by the movements of left hip, in particular by abduction and external rotation. Moreover, pain did not allow him to put weight on his left hip. However, no other abnormalities were found. At that time he was initially diagnosed as having hip arthritis. Complete blood cell count showed $4400 / \mathrm{mm}^{3}$ white cells $\left(\right.$ CD4 $\left.96 / \mathrm{mm}^{3}\right)$, and $574000 / \mathrm{mm}^{3}$ platelets. The haemoglobin was $10.6 \mathrm{~g} / \mathrm{dl}$. Erythrocyte sedimentation rate was $114 \mathrm{~mm} 1 \mathrm{st} \mathrm{h}$. No abnormality of other laboratory parameters was found. Blood cultures were negative.

\section{Radiological findings}

An anteroposterior radiograph of pelvis was normal (fig 1).

Sonography of the hip did not show fluid collection.

Computed tomography (CT) showed calcification of the ischiogluteal bursa without synovial fluid and swelling of obturator internus muscle (fig 2). Fine needle aspiration of the obturator internus muscle and the ischiogluteal bursa guided by CT only yielded a scarce amount of bloody material. Cultures of this material were negative. Not enough material was available for histological study. Treatment

Infectious Division

J Corredoira

Radiology Division M Vázquez-Caruncho

Hospital Xeral-Calde Lugo, 27004 Lugo, Spain

Correspondence to: Dr Miguel A González-Gay, Division of Rheumatology, Hospital Xeral-Calde, c Dr Ochoa s/n, 27004 Lugo, Spain.

Accepted for publication 7 December 1998

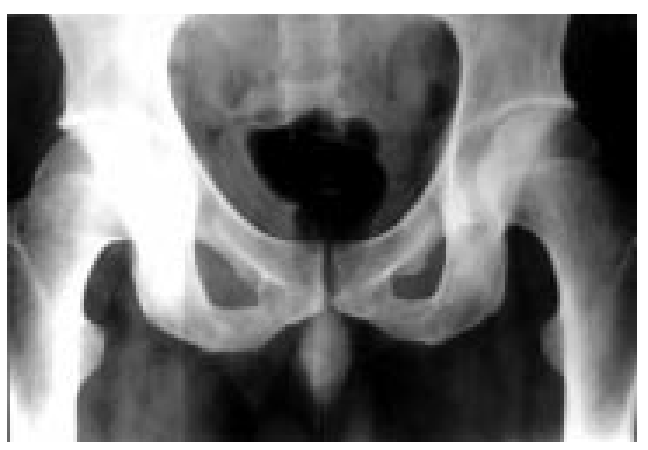

Figure 1 Anteroposterior radiograph of pelvis was normal.

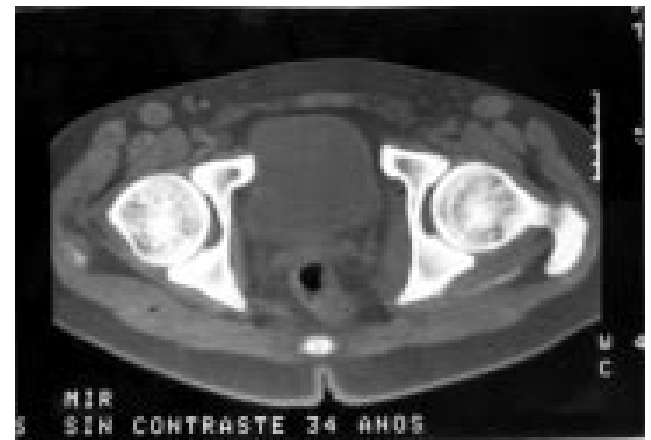

Figure 2 CT showed calcification of ischiogluteal bursa and swelling of obturator muscle.

with non-steroidal anti-inflammatory drugs (indomethacin $150 \mathrm{mg} /$ day orally) yielded a dramatic improvement of symptoms. Three weeks after the commencement of the antiinflammatory treatment the patient was asymptomatic.

\section{Diagnosis}

Obturator internus myositis and ischiogluteal bursitis.

\section{Discussion}

Focal myositis is a benign inflammatory disorder that may have problems of differentiation from other disorders such as infections or tumours. ${ }^{1}$ The aetiology of focal myositis is unknown and the course is generally one of regression. History of trauma and evidence of systemic symptoms are usually absent. In general, serum muscle enzymes and other routine laboratory parameters are normal. Histological features show severe inflammatory myopathy with necrosis, inflammation and later focal fibrosis. ${ }^{1}$ The differential diagnosis includes, when single, a muscle tumour (sarcoma or rhabdomyosarcoma) or proliferative fasciitis and myositis and, when multiple, muscle infarcts such as can occur in polyarteritis nodosa.

Pain in the hip region may arise from the hip joint itself, adjacent bones or periarticular soft tissues (cutaneous and subcutaneous tissues, muscles, tendons and bursas). In addition, thoracolumbar spine disorders, intraabdominal pathologies and peripheral vascular diseases can present with referred pain in this region and should therefore be considered in the differential diagnosis.

The obturator internus muscle originates from the anterolateral wall of the pelvic cavity around most of the obturator foramen. Its 
fibres converge acutely toward the lesser sciatic foramen, ending in a tendinous band that passes posteriorly across the posterior capsule of the hip joint. It then fuses with the gemelli and attaches on the greater trochanter's medial surface. ${ }^{2}$ In our case the radiological criteria of myositis involving the obturator internus muscle were met. Linear calcifications were seen with CT along the path of the muscle. Calcifications of the muscle occur more commonly in myositis ossificans than in inflammatory myositis. Indeed, in adults with inflammatory myositis calcifications are rare. CT performed 14 days later did not show evident calcification nor ossification in the rest of the muscle. With this in mind, CT was considered for three months later. However, regrettably, the patient was lost for follow up. On the other hand the occurrence of calcification in bursitis has also been previously described, especially in the subdeltoid and trochanteric bursas. Inflammation of the many bursas of the body is a common cause of painful musculoskeletal syndrome. ${ }^{3}$ The causal relation between bursitis and trauma has been well recognised. The ischial or ischiogluteal bursa is a deep bursa situated over the bony prominence of the ischium; inflammation of this bursa causes discomfort with sitting ("weaver's bottom"). ${ }^{4}$ Ischiogluteal bursitis may be more common than is generally considered as both the above mentioned conditions and others such as lumbosacral strain or even thromboflebitis may mimic this condition. The ischiogluteal bursa overlies the sciatic and the posterior femoral cutaneous nerves. For this reason, the pain may radiate and a misdiagnosis may be easily made. In general, palpation over the ischial tuberosity should elicit significant pain. However, in some cases as in our patient, tenderness to palpation is not clearly limited to the ischiogluteal bursa itself and the occurrence of pain to palpation over several other areas of the hip may lead to confusion in the examiner and produce a delay in the diagnosis.

Since the onset of HIV epidemy a variety of rheumatological manifestations and autoimmune phenomena has been reported in these people. ${ }^{5}$ The frequency of different musculoskeletal manifestations varies greatly between different studies, ranging from $0.15 \%$ to $12 \%{ }^{6}$ Also, although pyomyositis and infections of the deep bursas are rare in these patients, unusual organisms have been involved. Deep pelvic infections must be distinguished from extension of septic arthritis with contiguous bursal involvement and usually may be seen in the setting of systemic infection with bacteraemia.
Immunocompromised patients do not seem to be at a greater risk of developing septic bursitis. ${ }^{7}$ In our case both the absence of positive cultures from the material obtained from the bursa and muscle and the improvement observed with non-steroidal anti-inflammatory agents ruled out an infectious disease.

A detailed history and careful physical examination are essential to reach a definitive and precise diagnosis. The analysis of fluid extracted from muscle and bursa may be of prime importance in the diagnosis of a possible infectious disease. Also, several imaging techniques are available to identify myositis and bursitis. Conventional radiographs are useful for associated bony pathology (fractures, osteomyelitis and underlying joint disease) and soft tissue abnormalities (calcification, swelling, and gas). CT has been used to study the anserine bursa, gastrocnemius-semimaembranosus bursa, and iliopsoas bursa. ${ }^{1}$ Both CT and magnetic resonance imaging have proved to be useful in studying focal myositis as they may help to find the location and size of the mass as well as to provide a non-invasive method of follow up. ${ }^{8-10}$ In our case CT provided useful information about the cause of hip pain of the patient. Whenever a septic condition is excluded, a careful injection with local anaesthetic and corticosteroids in the bursa may be of great help to support the diagnosis and improve the symptoms of the patient. In our patient both the absence of positive cultures from the material obtained from the bursa and muscle and the improvement observed with non-steroidal anti-inflammatory agents ruled out an infectious disease.

1 Finger DR, Dennis GJ. Focal myositis. J Rheumatol $1995 ; 22: 188-9$

2 Gray's anatomy of the human body. British ed 37. In: Williams PL, Warwick R, eds. Philadelphia: WB Saunders, 1988: 542-645.

3 Zimmermann B III, Mikolich DJ, Ho G Jr. Septic bursitis. Semin Arthritis Rheum 1995;24:391-410.

4 Swartout R, Compere EL. Ischio-gluteal bursitis. JAMA 1974;227:551-2.

5 Vassilopoulos D, Chalasani P, Jurado RL, Workowski K, Agudelo C. Musculoskeletal Infections in patients with human immunodeficiency virus infection. Medicine 1997; $76: 284-94$

6 Calabrese LH. Human immunodeficiency virus (HIV) infection and arthritis. Rheum Dis Clin North Am 1993;19:477-88.

7 Roschmann RA, Bell CL. Septic bursitis in immunocompromised patients. Am J Med 1987;83:661-5.

8 Moskovic E, Fisher C, Westbury G, Parsons C. Focal myositis, a benign inflammatory pseudotumor: CT appearances. Br J Radiol 1991;64:489-93.

9 Moreno-Lugris C, González-Gay MA, Sánchez-Andrade A, Blanco R, Basanta D, Ibañez D, et al. Magnetic resonance imaging: a useful technique in the diagnosis and follow-up imaging: a useful technique in the diagnosis and

10 Fraser DD, Frank JA, Dalakas M, Miller FW, Hicks JE, Plotz P. Magnetic resonance imaging in the idiopathic inflammatory myopathies. J Rheumatol 1991;18:1693700 . 